Vorarbeit 1: Himmelwärts - Zauberposse in sieben Bildern: Merkl 

\|(I.) Sport.

Einweihung eines Ruderbootes.

DER PRÄSIDEnT Fahre hin! Eile von Sieg zu Sieg! Wie $\ulcorner$ Dein $\urcorner$ stolzer Vorfahre, der leider zerschellt ist! Fahre hin und bekomme den Namen Amititia! Von dem Verein der $\ulcorner$ kaufmännischen $\urcorner$ Angestellten der Union-Bank! Ein $\ulcorner$ Hurrah $\urcorner$ auf unser Vaterland!

AlLE $\ulcorner$ Hurrah! Hurrah! Hurrah!

(Musik)

KASIMIR Was hat denn das Vaterland mit ${ }^{\ulcorner}$dem Faltboot ${ }^{\urcorner}$zu tun?

10

Der Merkl Franz Fräulein! Ich muss mit Ihnen etwas Wichtiges reden unter vier Augen: der Vorstand will es nicht haben, dass Sie diesen Herren da mitgebracht haben! Der Herr ist ein Arbeiter - und Sie kennen unsere Prinzipien, dass wir uns streng gegen die Arbeiterschaft absondern -\ulcorner\urcorner

15 Karoline Aber das ist doch mein Bräutigam!

Der Merkl Franz Egal! Wir können hier verwandtschaftliche Beziehungen nicht dulden!

\Abbruch der Bearbeitung\

\begin{tabular}{rll}
\hline 3 & $\ulcorner$ Dein $\urcorner]$ & {$[\{\mathrm{d}\}] \mid$ D|ein } \\
5 & $\ulcorner$ kaufmännischen $\urcorner]$ & korrigiert aus: |kaufmännisch/ \\
5 & $\ulcorner$ Hurrah $\urcorner]$ & {$[\{$ Hoch $\}] \mid$ Hurrah| } \\
7 & $\ulcorner$ Hurrah $\rightarrow$ Hurrah! $\urcorner]$ & {$[$ Hoch! Hoch! Ho] $\mid$ Hurrah $\rightarrow$ Hurrah!| } \\
9 & $\ulcorner$ dem Faltboot $\urcorner]$ & d[e \{\}$] \mid$ em| [B] |Faltboot $\mid$ \\
10 & \ulcorner\urcorner$]$ & {$[$ KAROLINE $($ zu Kasimir $)$ Was machst denn für ein fades Gesicht?] } \\
14 & \ulcorner\urcorner$]$ & [- Ich bitte Sie aber $]$
\end{tabular}


Strukturpläne

ÖLA 3/W 50 - BS 41 a, Bl. 2

Tun Hinumd.

Zainhreme mit gesang ind Tawas.

1.) Dí Denkmalsmathilling.

2x) Im thinemd: Landeplater.

Ein Feder den den Zurilas

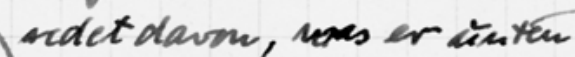
vereanon lat:

Whimus clefrass Nichts. Och herme ming maine Wirsens ceafe sanst midat. Deh his cile Demelomicis.

Jch hale lain Vortar id

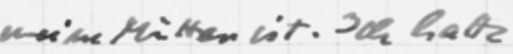
mins sin riàde, ales die hiunt ich schan urpalmarrom - dis ist

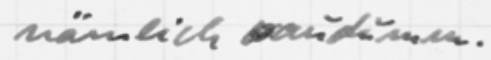

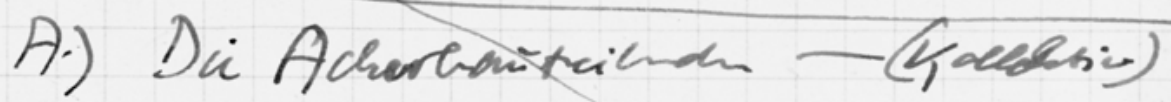

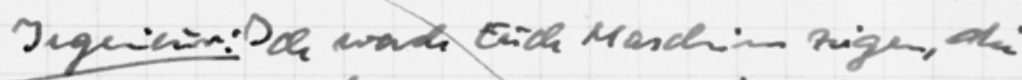
rude grassain - vicrengross -

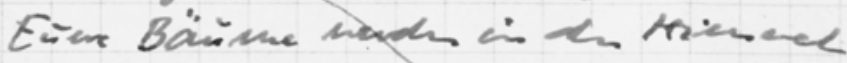
machsen!

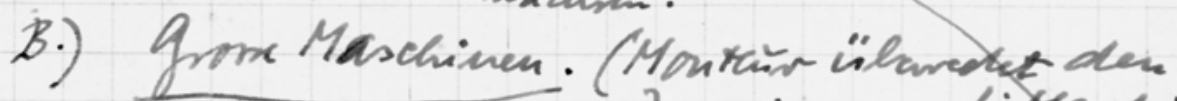

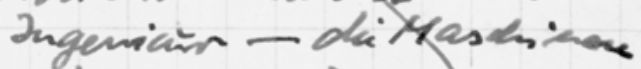

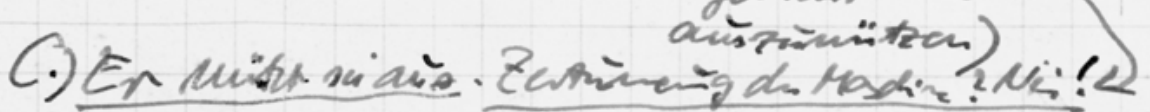

28 


\section{Im Himmel.}

Zauberposse mit Gesang und Tanz.

\section{Personen:}

1.) Die Denkmalsenthüllung.

2.) Im Himmel: Landeplatz.

Ein Jeder von den Zweien Zweien redet davon, was er unten

[Bitte. Los -] verlassen hat:

Monteur: Wallen Sie mir nicht von dem grossen Mann erzählen?

4 [Erinnerung an den grossen Mann Blumenstrauss

(auf dom Sockel)

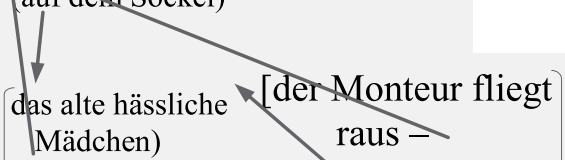

Mädchen)

\section{Erster:}

Wissenschaftler: Nichts. Ich kenne nur meine Wissenschaft sonst nichts. Ich bin eine Doppelwaise.

Monteur: Ich habe keinen Vater und meine Mutter ist. Ich hatte nur ein Mädel, aber die könnt ich schon verschmerzen - die ist nämlich saudumm.

A.) Die Ackerbautreibenden - (Kollektiv)

Ingenieur: Ich werde Euch Maschinen zeigen, die werden gross sein - riesengross Euere Bäume werden in den Himmel wachsen!

B.) Grosse Maschinen. (Monteur überredet den Ingenieur - die Maschinen auszunützen)

C.) Er nützt sie aus. Zertrümmerung der Maschine? Nein! 


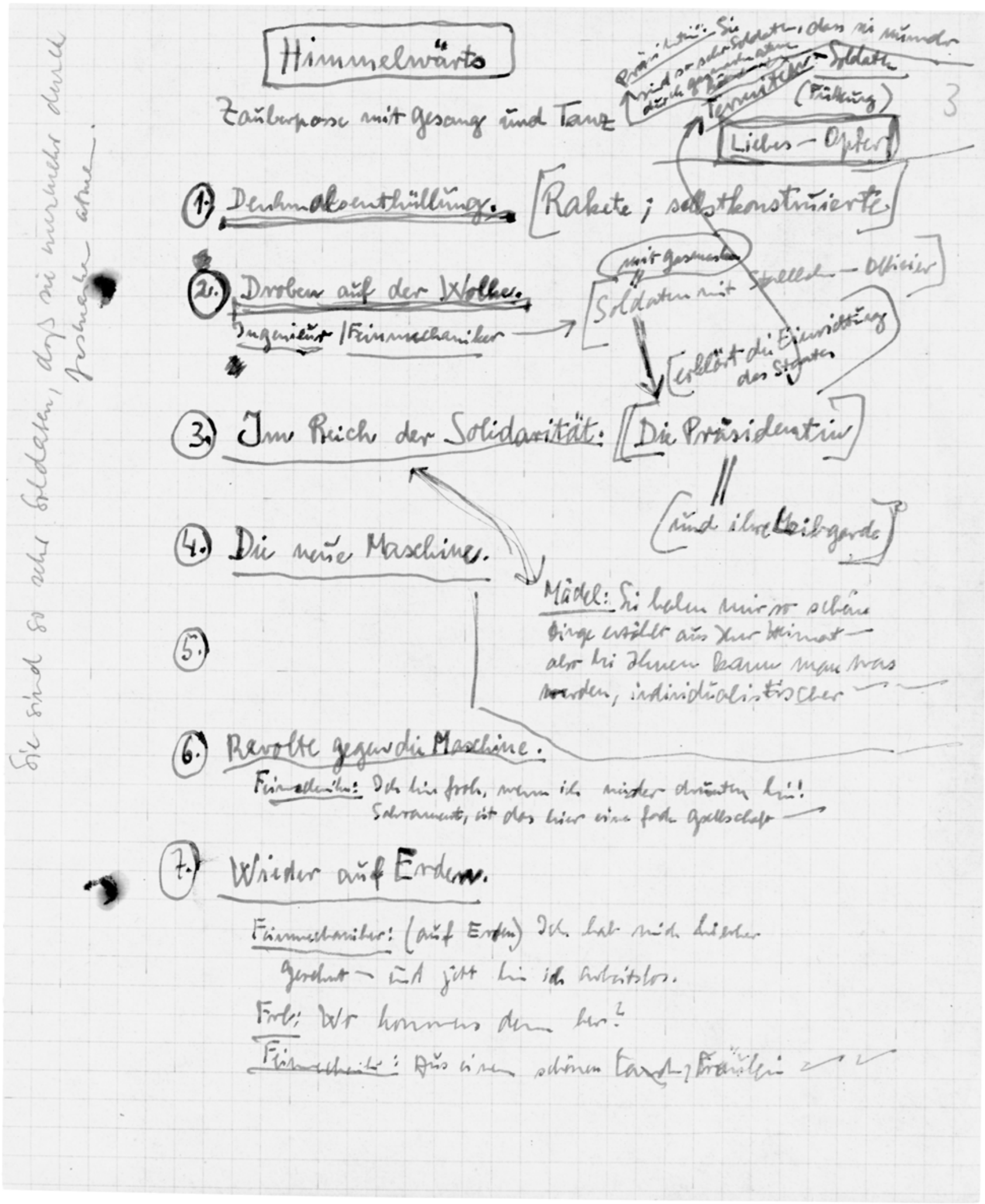




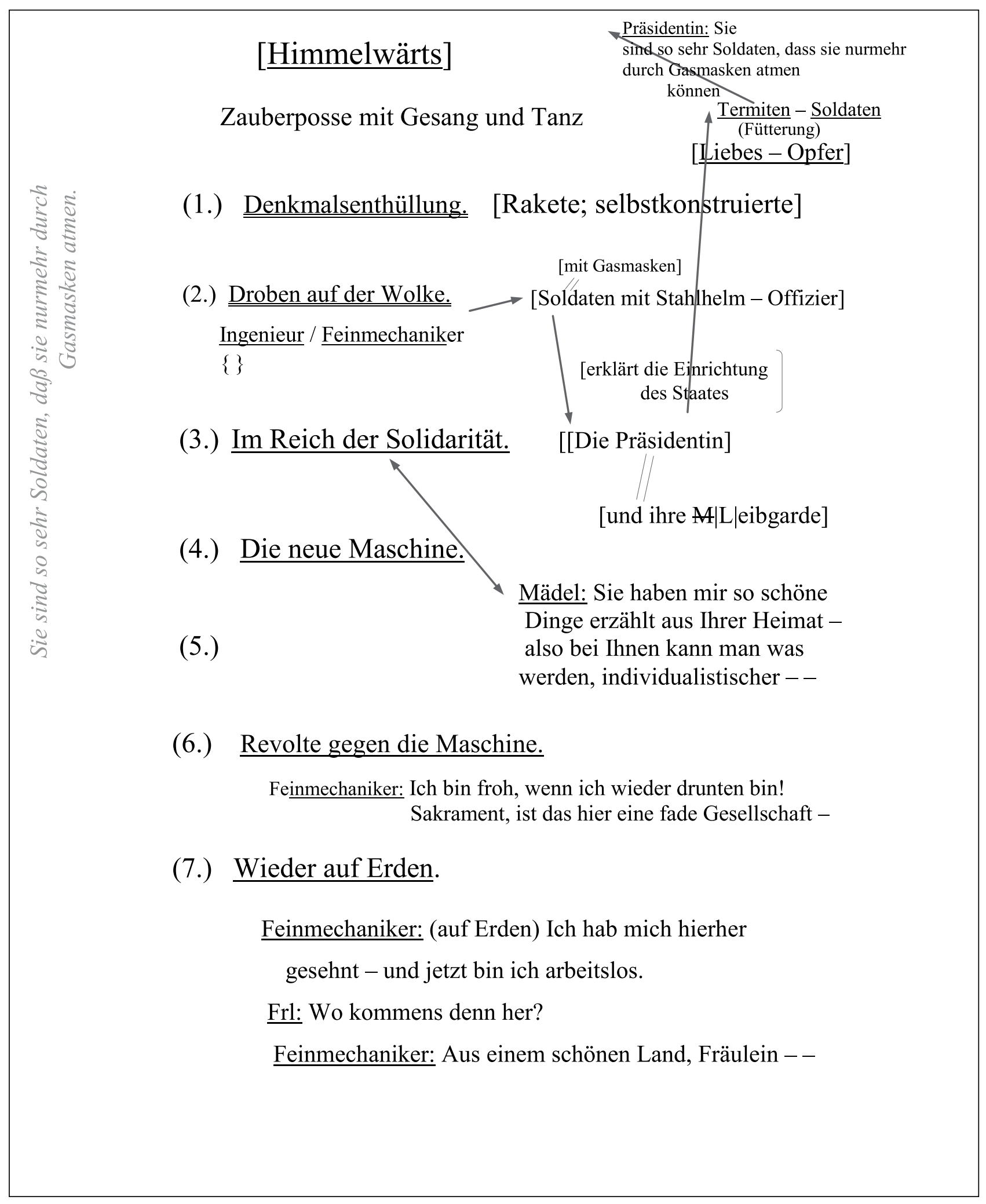




\section{L}

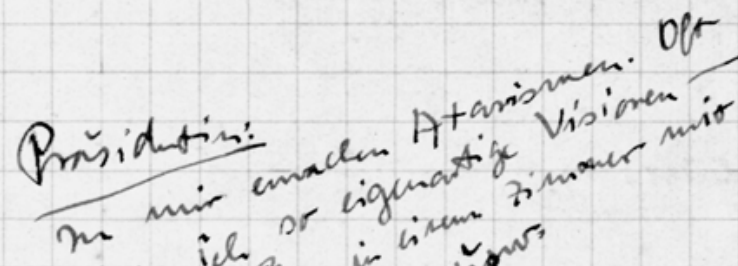

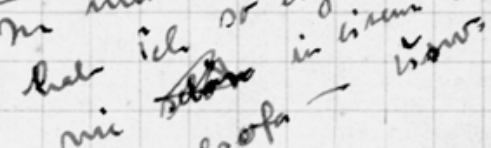

$$
\begin{aligned}
& \text { plin }
\end{aligned}
$$




\section{Präsidentin:}

In mir erwachen Atavismen. Oft hab ich so eigenartige Visionen wie schön in einem Zimmer mit

Plüschsofa - usw. 


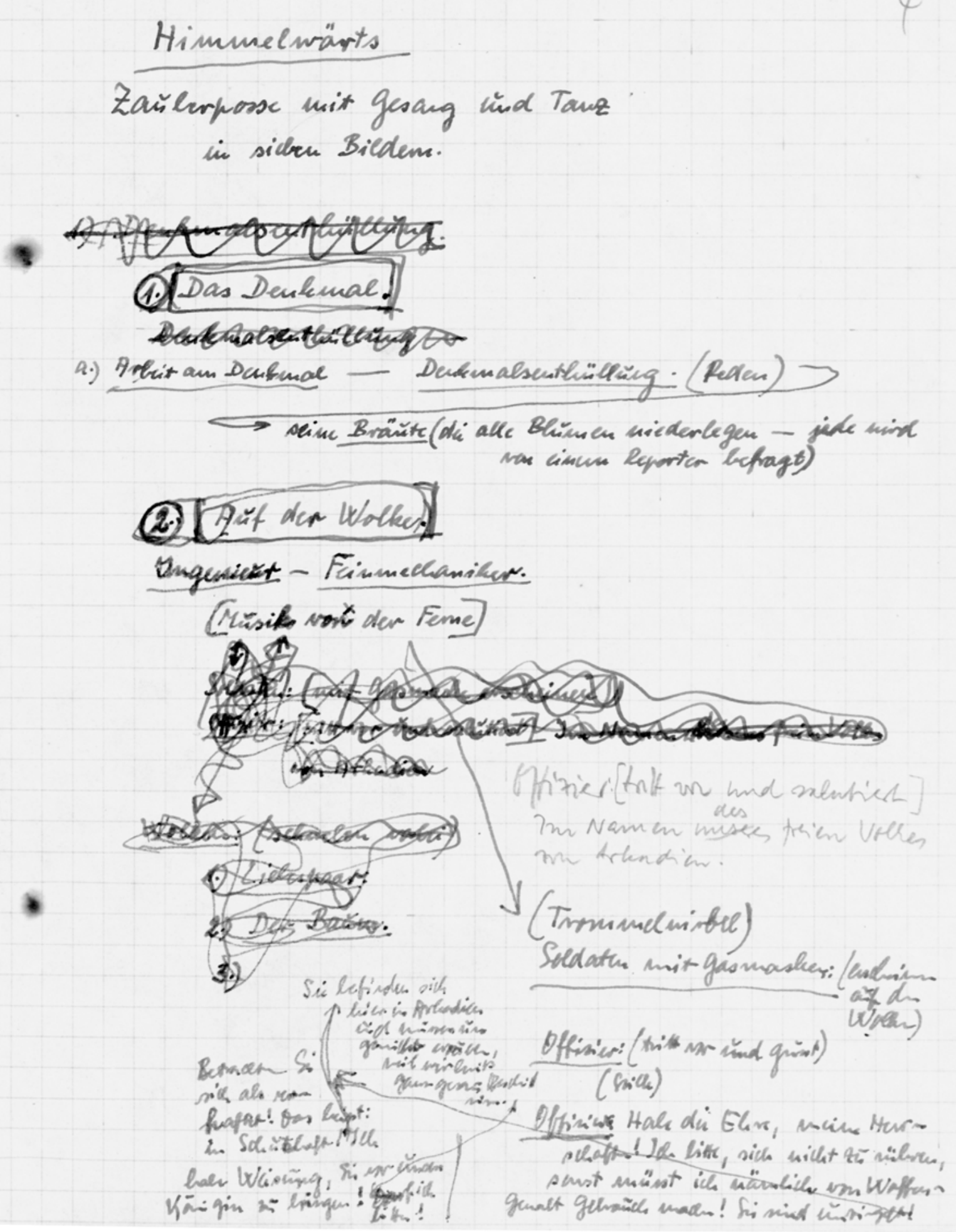




\section{$\underline{\text { Himmelwärts }}$}

\section{Zauberposse mit Gesang und Tanz}

in sieben Bildern.

\section{1.) Denkmalsenthüllung.}

\section{(1.) [Das Denkmal.]}

Đenkmalsenthüllung -

a.) Arbeit am Denkmal - Denkmalsenthüllung. (Reden)

$\leftarrow$ seine Braute (die alle Blumen niederlegen - jede wird von einem Reporter befragt)

\section{(2.) [ Auf der Wolke.]}

Ingenieur - Feinmechaniker.

[Musik von der Ferne]<smiles>[CH]1C=C1</smiles>

Soldaten: [mit Gasmasken erseheinen]

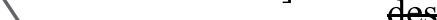

Oeffizier: [tritt vor und salutiert] Im Namen unseres freien Volkes

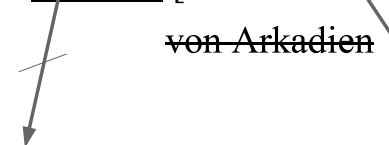

Wolken. (schweben vorbei)

1.) Liebespar.

2.) Der Baum.

3.) Sie befinden sich

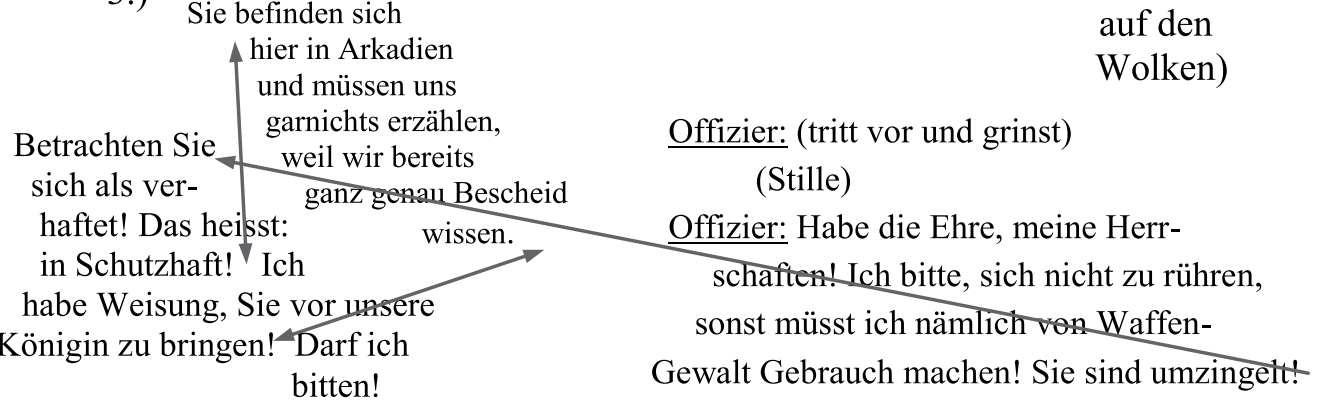




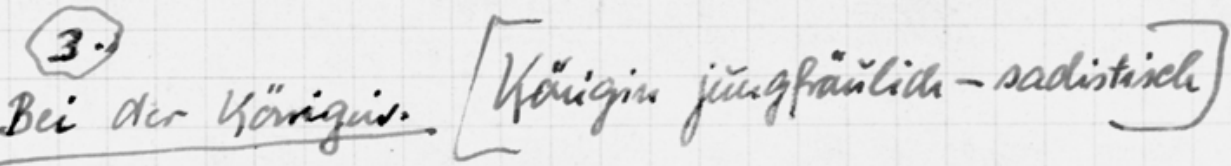

(4-)

Bider Arecix.

(5.)

Bcim Tanz.

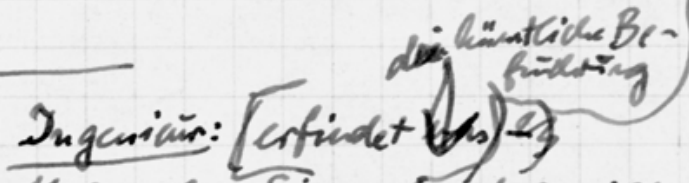
Mchanviluen: Sa marden doch wicht sriblid ocion, dan Siidlas deven gchen - okme Geganleriting.' Sis kinumer König werden, his motens die Yörigin innd - Solhin!

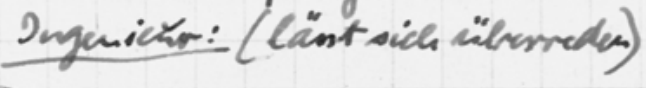

\footnotetext{
(6.)

Protest der Eracien. [Geribat über den Mechaniluen]

7. Denlemial.
} 
(3.)

Bei der Königin. [Königin jungfräulich - sadistisch]

Bei der Arbeit.

(5.)

Beim Tanz. Ingenieur: [erfindet was) $\rightarrow$ die künstliche BeMechaniker: Sie werden doch nicht so blöd sein, dass Sie das denen geben - ohne Gegenleistung! Sie können König werden, heiratens die Königin und - Schluss! Ingenieur: (lässt sich überreden)

(6.)

Protest der Frauen. [Gericht über den Mechaniker]

(7.) Denkmal. 


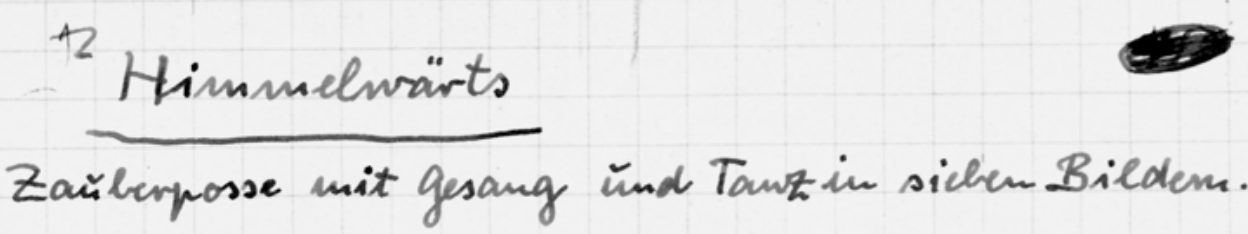

1. Bild: Denkmalenthin Uinging.

2. Bild: $\frac{\text { Aüf der Wrake. }}{\frac{\text { Soldatew. }}{\text { Palast de Königin. }}}$

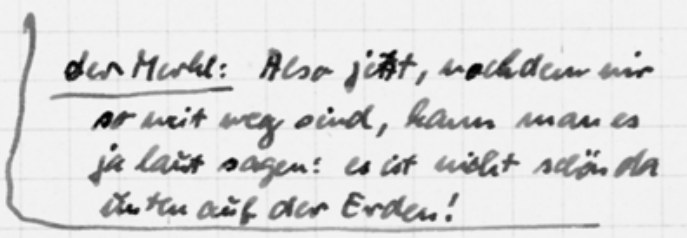

3. Bild: Vor deun Haüse des Herm Sohivininger

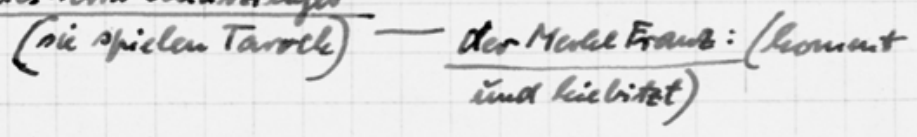

4. Bild:- Alle Whiler: Es ist cin fremder angehommen - tien

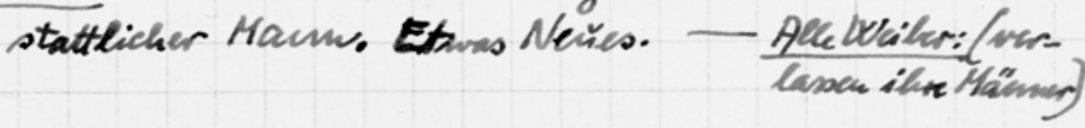

5.Bild: Dngenieñ - Konigin.

6. Bied: Das Fest.

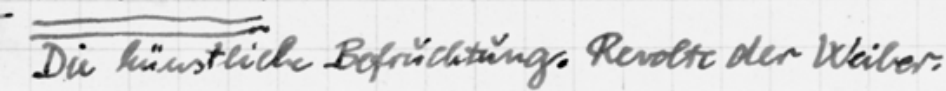

7. Bild: Beim Denkenal. 


\section{Himmelwärts}

Zauberposse mit Gesang und Tanz in sieben Bildern.

1. Bild: Denkmalenthüllung.

$\circledast$

2. Bild: Auf der Wolke.

Soldaten.

Palast der Königin.

$\circledast$

der Merkl: Also jetzt, nachdem wir so weit weg sind, kann man es ja laut sagen: es ist nicht schön da unten auf der Erden!

3. Bild: Vor dem Hause des Herrn Schürzinger

(sie spielen Tarock) - der Merkl Franz: (kommt und kiebitzt)

[4. Bild:] Alle Weiber: Es ist ein Fremder angekommen - ein stattlicher Mann. Etwas Neues. - Alle Weiber: (verlassen ihre Männer)

5. Bild: Ingenieur - Königin.

\section{Bild: Das Fest.}

Die künstliche Befruchtung. Revolte der Weiber.

\section{Bild: Beim Denkmal.}


Strukturplan in sieben Bildern

ÖLA 3/W 50 - BS 41 a, Bl. 17v

(1.) Denknaluthiseling.

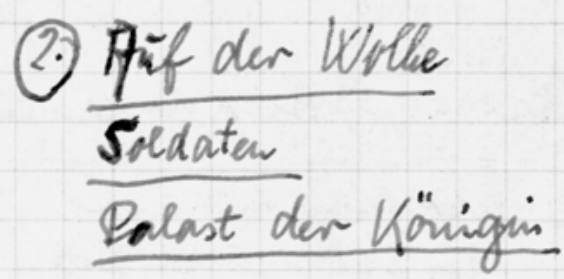

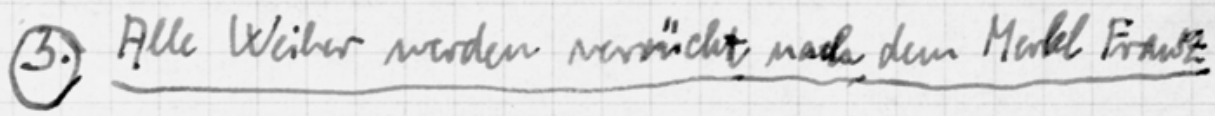

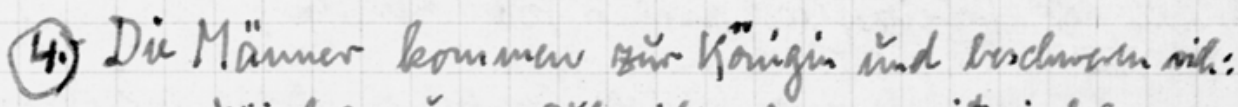

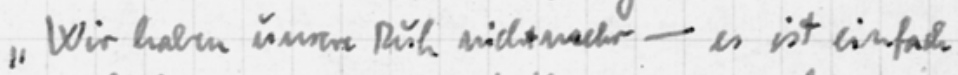

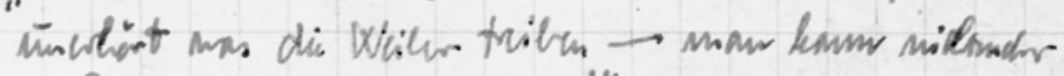

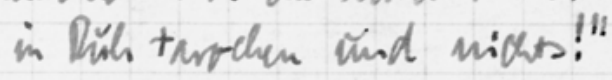

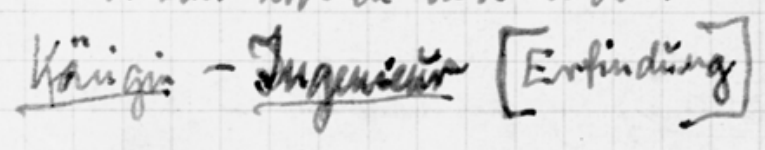

5.) Vorbsuredisingy mür Ruots.

6.) Das Fest.

(9) Beim Dentemal.

40 
(1.) Denkmalenthüllung.

(2.) Auf der Wolke

Soldaten

Palast der Königin

(3.) Alle Weiber werden verrückt nach dem Merk1 Franz

(4.) Die Männer kommen zur Königin und beschweren sich:

„Wir haben unsere Ruh nichtmehr - es ist einfach unerhört was die Weiber treiben - man kann nichtmehr in Ruh tarocken und nichts!“”

$\underline{\text { Königin }}$ - Ingenieur [Erfindung]

(5.) Vorbesprechung zur Revolte.

(6.) Das Fest.

(7.) Beim Denkmal . 


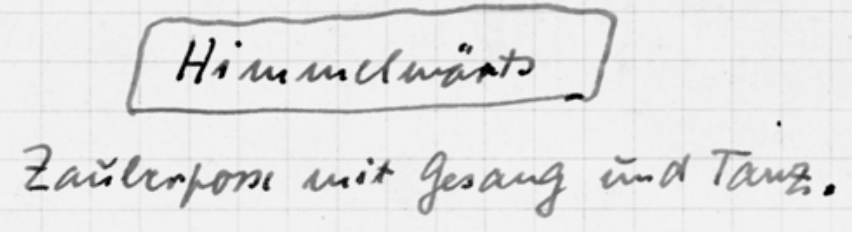

1.) Denlcumalscuthinllingng:

2.) Gelandet in Arkadicu.

Juguin - Fuimechailus - Sclaten - Offirter.

(Dë Wothentilew sich: Palast dex Königin)

dic Königies - Gefolge: Gunemal

Q

A.) Ingenicür: (erfindet dic kïnstlicke Beforichtieng)

B.) Feinmechaniber: (Organisiert den Arufstande der

Fravien Gegen dic kisustlich Bopinalivingng

[Rever]

C.) Niederschlagring der Revolte (diench dic Königin)

(4)

7.) Wieder unten ann. Denlemal. 


\section{[Himmelwärts]}

Zauberposse mit Gesang und Tanz.

1.) Denkmalsenthüllung.

2.) Gelandet in Arkadien.

Ingenieur - Feinmechaniker - Soldaten - Offizier.

(Die Wolken teilen sich: Palast der Königin)

die Königin - Gefolge: General

Mitarbeiterinen.

$\circledast$

A.) Ingenieur: (erfindet die künstliche Befruchtung)

B.) Feinmechaniker: (organisiert den Aufstand der Frauen gegen die künstliche Befruchtung $\longrightarrow$

$\longleftarrow$ die Frauen wählen ihn zu ihrem König)

$\circledast \quad$ Revolte]

C.) Niederschlagung der Revolte (durch die Königin)

$\circledast$

$(\boxplus|7|$.$) Wieder unten am Denkmal.$ 


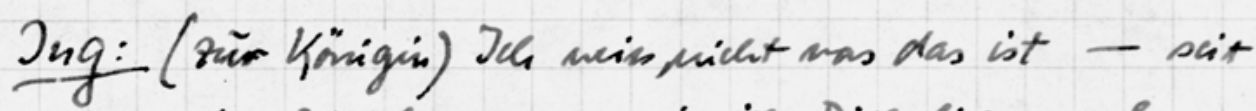 ich Dich kenne - scit ich Dich liche - baum

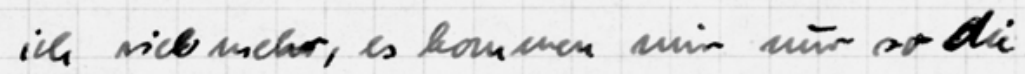 Jodeen - añch dic kinstliche Befrieltäning.}


Ing: (zur Königin) Ich weiss nicht was das ist - seit ich Dich kenne - seit ich Dich liebe - kann ich viel mehr, es kommen mir nur so die Ideen - auch die künstliche Befruchtung. 


\section{Himmel wairts}

Zaiberpose mit gesang ind Tawz.

1.) Denkmabenthilling der Mane, der sohuandest

2.) Widke - Palest

3.) Die Mämer sind Spiesser. Die Wiler híngerige: 1. Pear
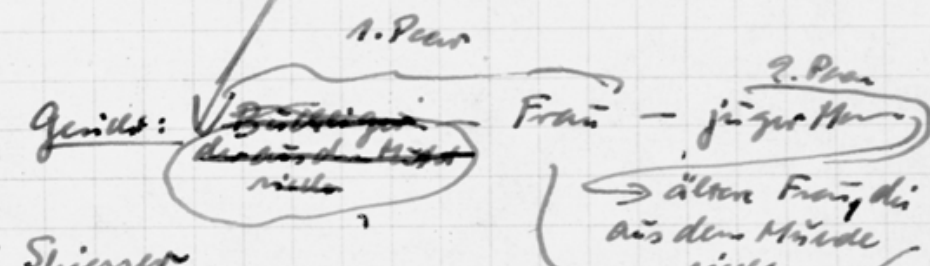
ass den Mrinde nicus.

4.) Dir Mäumer besdawen side. (Erfindinge)

5.) Das Fest.

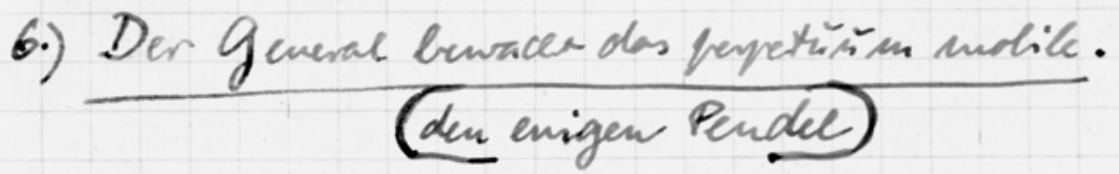

7.) Denkmak. 


\section{Himmelwärts}

Zauberposse mit Gesang und Tanz.

1.) Denkmalsenthüllung

2.) Wolke - Palast

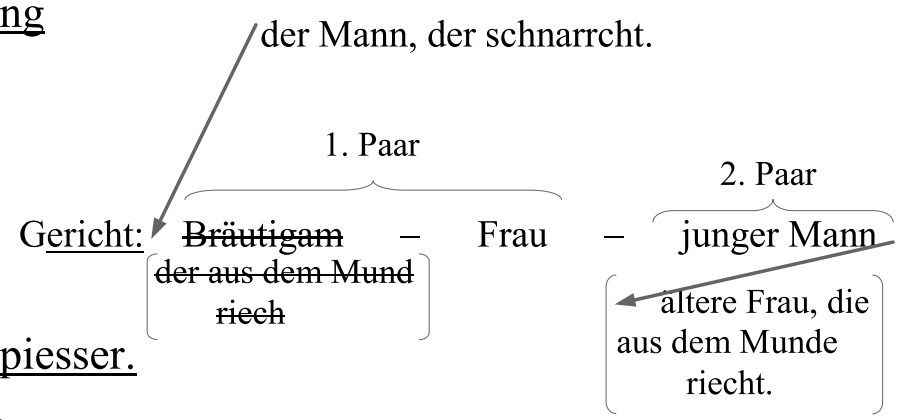

Die Weiber hungerig.

4.) Die Männer beschweren sich. (Erfindung)

5.) Das Fest.

6.) Der General bewacht das perpetuum mobile.

(den ewigen Pendel)

7.) Denkmal. 


\section{R}

1.) Denkmal.

2.) Wouc - Palast:

3.) Der General benacht das perpectünàn mobile

\section{4.) 4.2000}

5.)

6.)

7.) Denkmal.

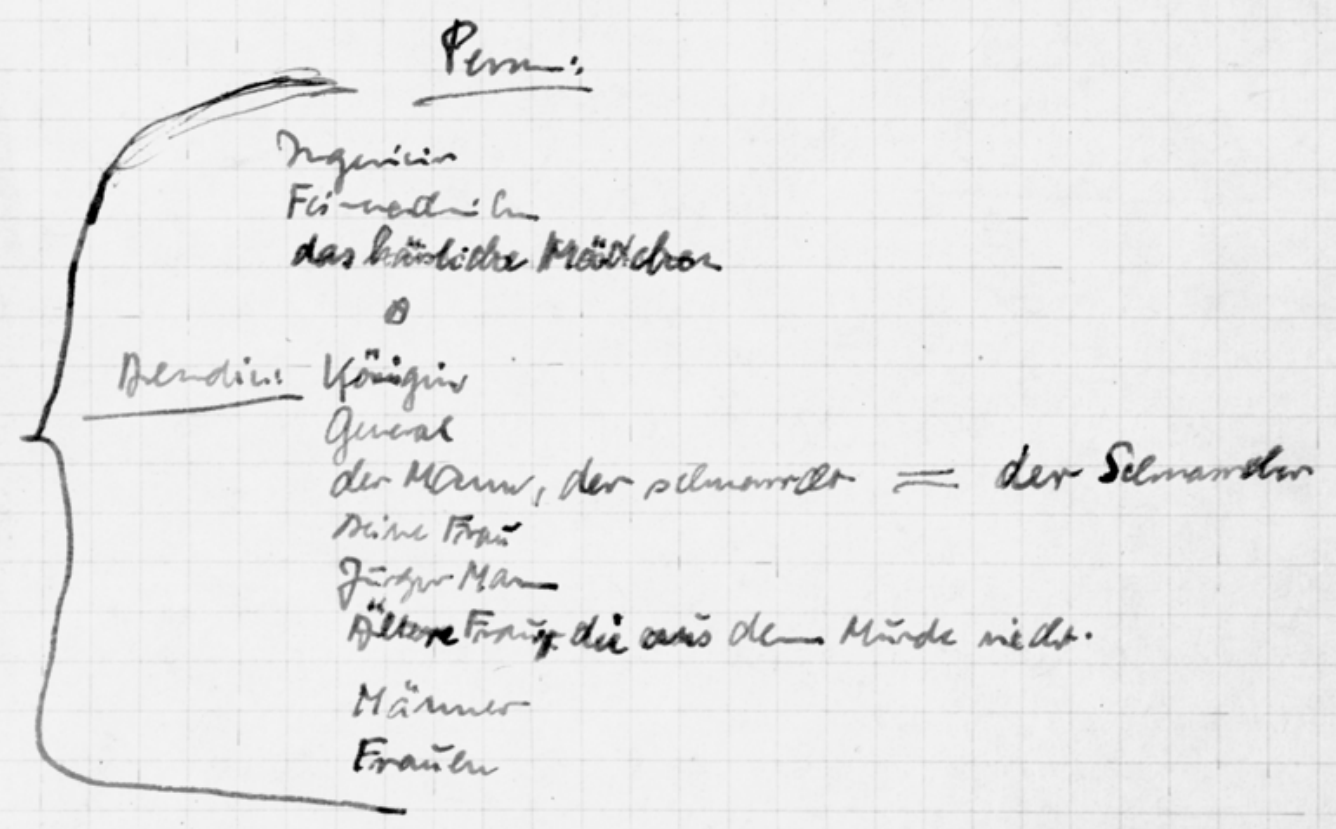




\section{1.) Denkmal.}

2.) Wolke - Palast.

3.) Der General bewacht das perpetuum mobile

\section{4.) Die Männer sind Spiesser.}

5.)

6.)

7.) Denkmal .

\section{Personen:}

\section{Ingenieur}

Feinmechaniker

das hässliche Mädchen

$\circledast$

Arkadien: Königin

General

der Mann, der schnarrcht $=$ der Schnarrcher seine Frau

Junger Mann

Ältere Frau, die aus dem Munde riecht.

Männer

Frauen 
Strukturplan, Notiz, Werktitel

ÖLA 3/W 50 - BS 41 a, Bl. 14

Himmelwärts

Zainberposse mit gesang ind Tawz.

(1.) Dentemal.

2.) Wollow- Polast.

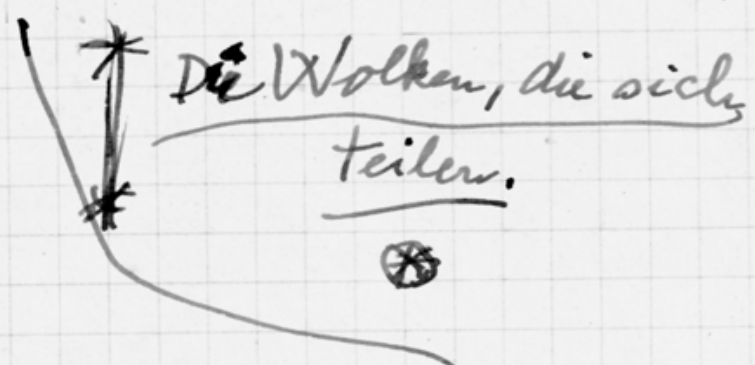

3.) Den Mechaniker gehts güt.

(Alle Frañen schuöirmen fïn ilun

(4.) Der Pendel.

5.) Das Fest.

(6.) Himmelwärts

$$
\text { Zainkorparse mit Gesoug àd Tous }
$$

(7.) Denkmal.

50 


\section{$\underline{\text { Himmelwärts }}$}

Zauberposse mit Gesang und Tanz.

$\circledast$

(1.) Denkmal.

Die Wolken, die sich teilen.

(2.) Wolke $-\underline{\text { Palast. }}$

$\circledast$ [Gericht] $\left|E^{14}\right|$

(3.) Dem $\{$ Mechaniker gehts gut. (Alle Frauen schwärmen für ihn)

(4.) Der Pendel .

(5.) Das Fest. $\underline{\text { Himmelwärts }}$

(6.) Zauberposse mit Gesang und Tanz. $\left|E^{15}\right|$

(7.) Denkmal.

$\left|E^{13}\right|$ 

Himsachärts.

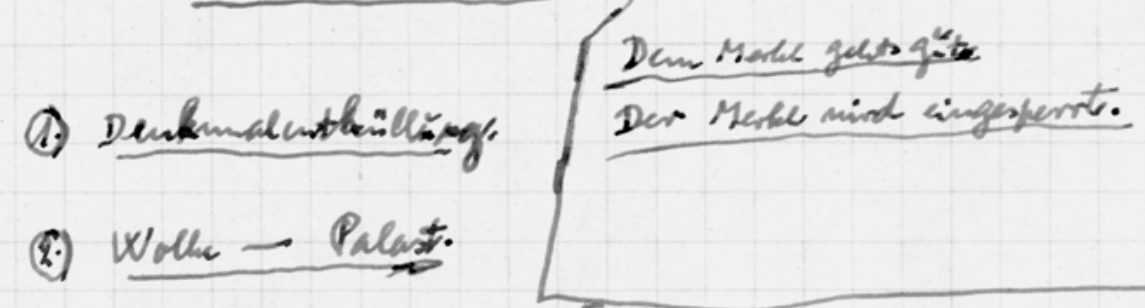
(3.) Desw Markl gelts gint:
Er

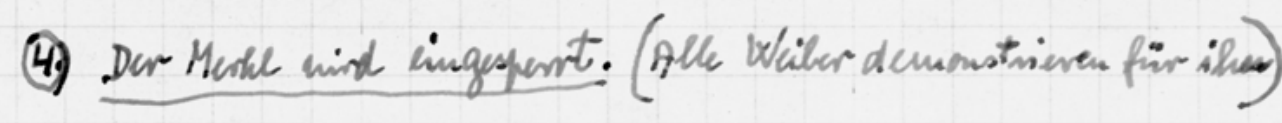

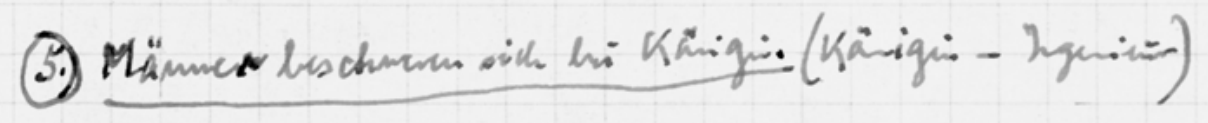

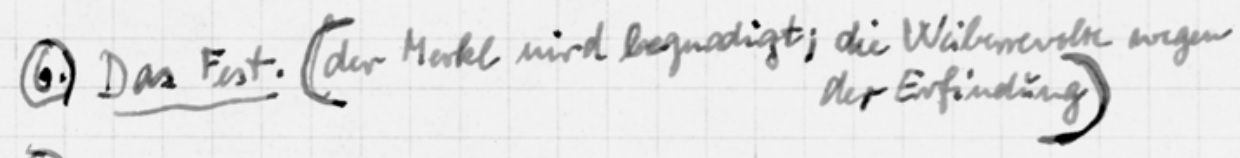
(7.) Denknal,

(2)

\section{Himunel wärts}

Pranklerpose mit gesang ind Taut is sicben Bildem an Od"so. Horráth.
1.) Denfunalenthilling
2.) Wolkc - Palast
3.) Deun Merre gelits gät
4.) Alec Wiler schwärmen fior den Mertel
5.) Alle Mämmen buschwerar sick bxider Youngin
6.) Das Fest. Dic Enfindinge]
7.) Denkemal. 


\section{Himmelwärts.}

(1.) Denkmalenthüllung.

Dem Merkl gehts gut.

Der Merkl wird eingesperrt.

(2.) Wolke - Palast.

(3.) Dem Merkl gehts gut. (Er bespricht ein kapitalistisches Komplott)

(4.) Der Merkl wird eingesperrt. (Alle Weiber demonstrieren für ihn)

(5.) Männer beschweren sich bei Königin. (Königin - Ingenieur)

(6.) Das Fest. (der Merkl wird begnadigt; die Weiberrevolte wegen der Erfindung)

(7.) Denkmal.

$\circledast$

$\left|E^{16}\right|$

\section{Himmelwärts}

Zauberposse mit Gesang und Tanz in sieben Bildern von Ödön Horváth.

1.) Denkmalenthüllung

2.) Wolke - Palast

3.) Dem Merkl gehts gut

4.) Alle Weiber schwärmen für den Merkl

5.) Alle Männer beschweren sich bei der Königin

6.) Das Fest. [Die Erfindung]

7.) Denkmal . 
Strukturplan, Konfigurationsplan, Figurenliste

ÖLA 3/W 50 - BS 41 a, Bl. 9

Himuelwärts

Zainkerpose mit Gesang inad Tane

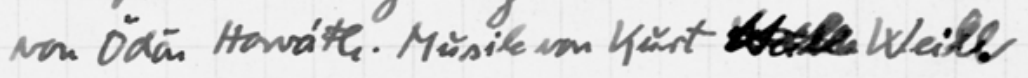

1.) Denkmalsentheillings.

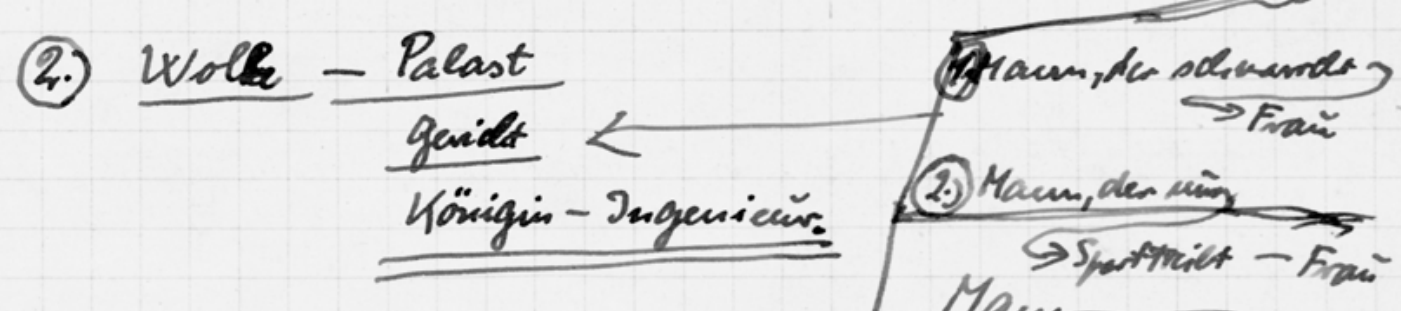

(3.)

(4.)

(5.)

(6.)

7.) Denkmal.

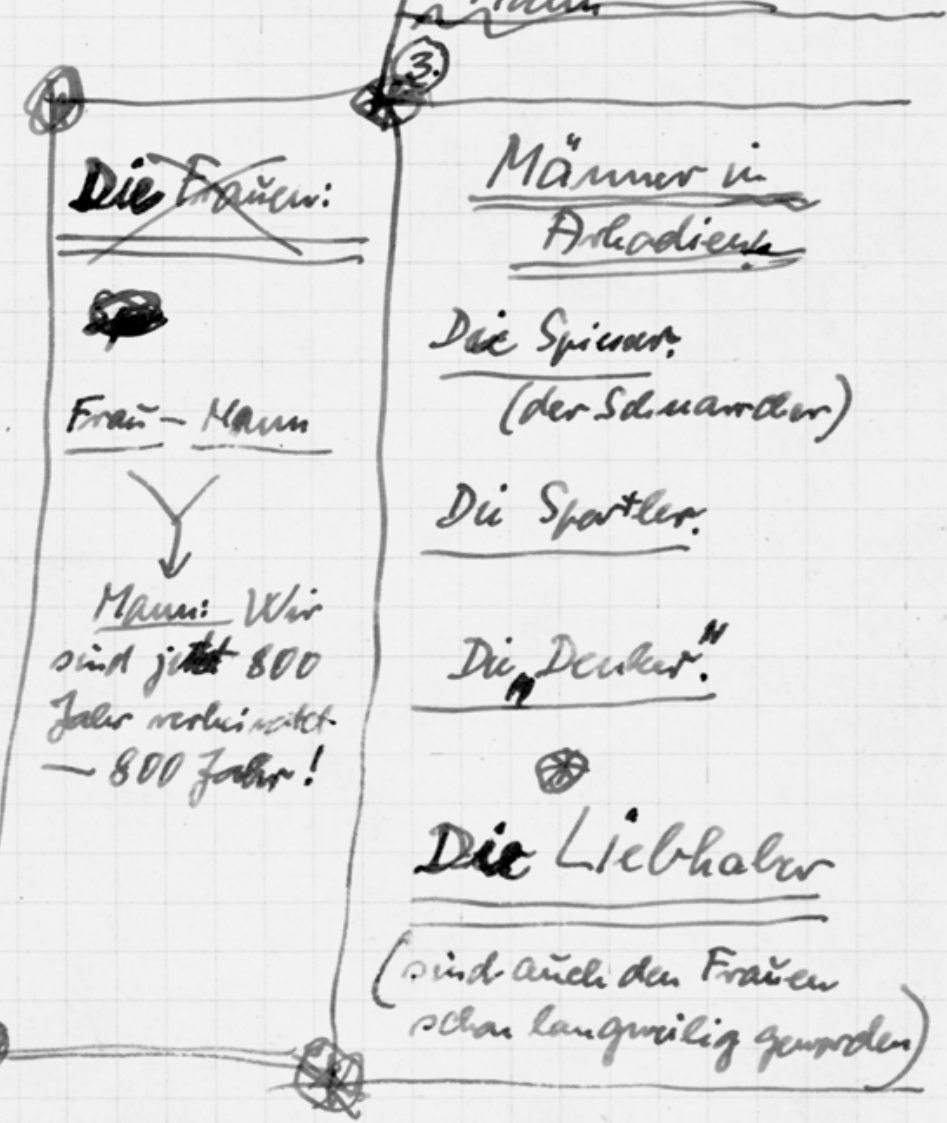

54 


\section{Himmelwärts.}

Zauberposse mit Gesang und Tanz

von Ödön Horváth. Musik von Kurt Weill Weil-|1|

(1.) Denkmalsenthüllung.

(2.) Wolke - $\underline{\text { Palast }}$

Gericht

Königin - Ingenieur.

(3.)

(4.)

(5.)

(6.)

(7.) Denkmal.

$\left|E^{19}\right|$

\section{Die Frauen:}

Sp<smiles>[C]1CC1</smiles>

Mann: Wir sind jetzt 800

Jahr verheiratet

$-800 \mathrm{Jahr}$ !
Frau - Mann
(1.) Mann, der schnarrcht Frau

(2.) Mann, der nur Sport treibt - Frau

(3.)

\section{Männer in}

Arkadien.

Die Spiesser. (der Schnarrcher)

Die Sportler.

Die „Denker“.

*

\section{Die Liebhaber}

$\left|E^{20}\right|$ schon langweilig geworden) $\mid E^{21} \backslash$ 
Dialogskizze, Replik, Strukturpläne

ÖLA 3/W 50 - BS 41 a, Bl. 13

II- Bild.

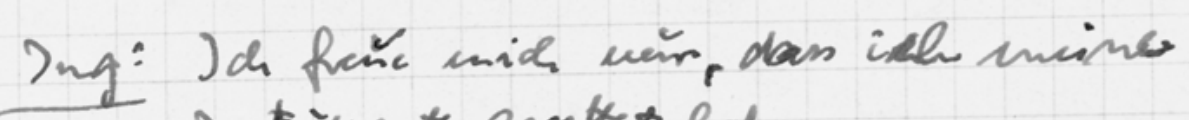
motinimente geoctert bab-

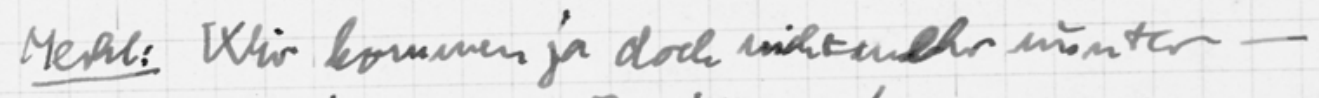
in: Jor Rithe maine Justimmente! Was näre ich phum hostimente - ?

III. Bild.

IV. Bild.

v. Bild.

Dु. Bild.

VII. Bild. Durkmal.
1.) Denhuar:

2.) Wolre - Palast

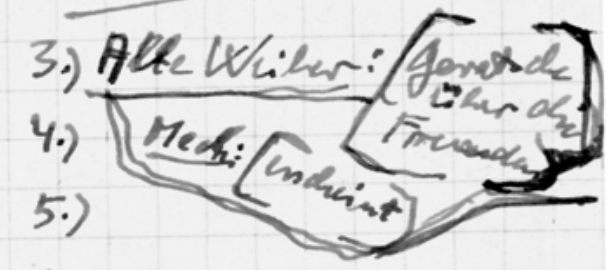
13 


\section{Bild.}

Ing: Ich freue mich nur, dass ich meine

Instrumente gerettet hab -

Merkl: Wir kommen ja doch nichtmehr runter -

Ing: Ich liebe meine Instrumente!

Was wäre ich ohne Instrumente? - - ? $\left.\quad\right|^{22}$

$$
\begin{aligned}
& \text { \{Ziel\} } \\
& \text { Ich bin \{hier droben\} Ingenieur } \\
& \begin{array}{lll} 
& \{\mathrm{Und}\} & \text { 1.) Denkmal. }
\end{array} \\
& \text { III. Bild. } \\
& \text { IV. Bild. } \\
& \text { 2.) Wolke-Palast } \\
& \text { 3.) Alle Weiber: Geratsche } \\
& \text { 4.) Mech: [erscheint] über die } \\
& \text { 5.) } \\
& \underline{\text { V. Bild. }} \\
& \text { 6.) } \\
& \text { 7.) } \\
& \text { VI. Bild. } \\
& \left|E^{25}\right| \\
& \text { VII. Bild. [Denkmal.] } \\
& \left|E^{24}\right|
\end{aligned}
$$


Dialogskizze und Notizen

ÖLA 3/W 50 - BS 41 a, Bl. 16

16

1.) Frainces: (Mreangen Einhilwing der Erfieding)

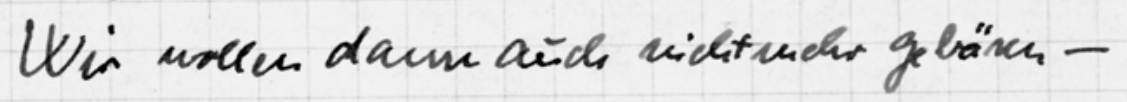

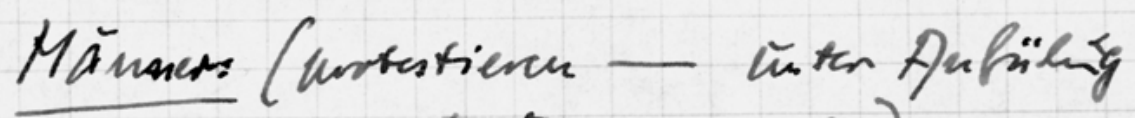
des Finmedhavilues)

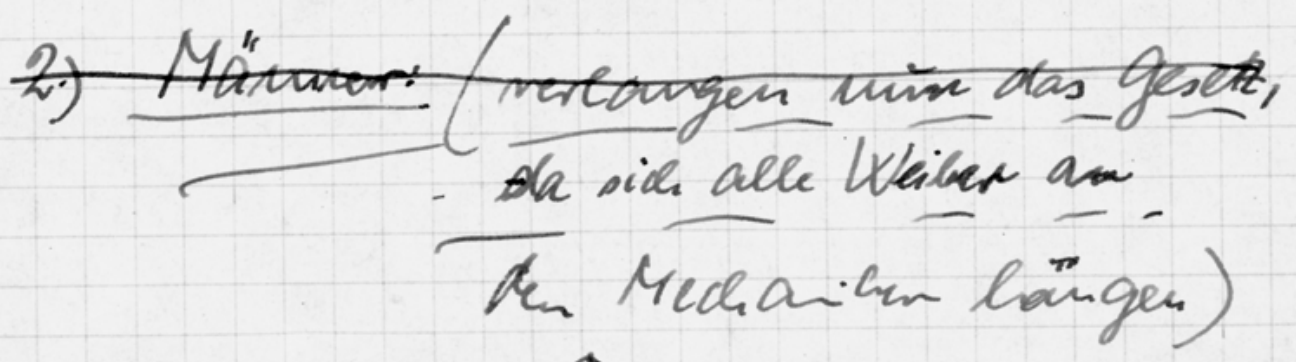

Mechanihet: (micgelt die Wriber ainf)

58 
1.) Frauen: (verlangen Einführung der Erfindung)

Wir wollen dann auch nichtmehr gebären -

Männer: (protestieren — unter Anführung des Feinmechanikers)

$\circledast$

z.) Männer:-_verlangennnundas_Gesetz.

da sich salle_Weiber an

den Mechaniker hängen)

$\circledast$

Mechaniker: (wiegelt die Weiber auf)

$\circledast$ 
Strukturplan in sieben Bildern

ÖLA 3/W 50 - BS 41 a, Bl. 17

Himnelwärts.

Zamberposse mit gesang ind Taws.

1.) Denlumal.

2.) Gclandet.

a.) Die Zwis:

a.) Sredaten

c.) Pacast den Yänigin .

Königin: Wir frieno kinte alend dive 27000 jäligh Bustand Gumsues Reiches! Daffich fitten ün Eviers Tilualuma!

3.) Bei der Arbeit. [Mccleaviluen frint sich an]

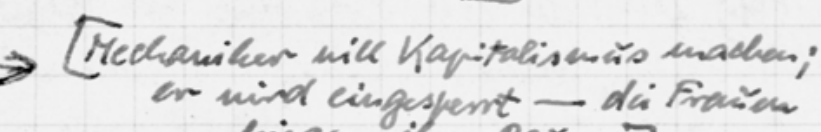

4.) Das Fest. enigan ilum Blininat

5.) Revolte der Fraüew. (gegaudii nover Enfindingng)

6.) Arsunising des Mechaviluero.

7.) Denkmal.

60 


\section{Himmelwärts.}

Zauberposse mit Gesang und Tanz.

1.) Denkmal.

2.) Gelandet.

a.) Die Zwei

b.) Soldaten

c.) Palast der Königin.

Königin: Wir feiern heute abend den 27000jährigen Bestand unseres Reiches! Darf ich bitten um Euere Teilnahme!

3.) Bei der Arbeit. [Mechaniker frisst sich an]

[Mechaniker will Kapitalismus machen; er wird eingesperrt - die Frauen

4.) Das Fest. bringen ihm Blumen]

5.) Revolte der Frauen. (gegen die neue Erfindung)

6.) Ausweisung des Mechanikers.

7.) Denkmal. 
Strukturplan in sieben Bildern

ÖLA 3/W 50 - BS 41 a, Bl. 12

Himmelwärts

Zainberrone mit Gesang unda Tanz.

1.) Denkmal

2.) Wolke

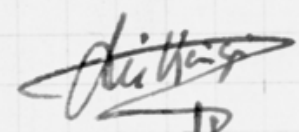

12

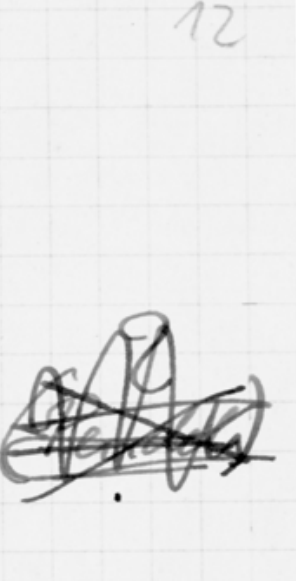

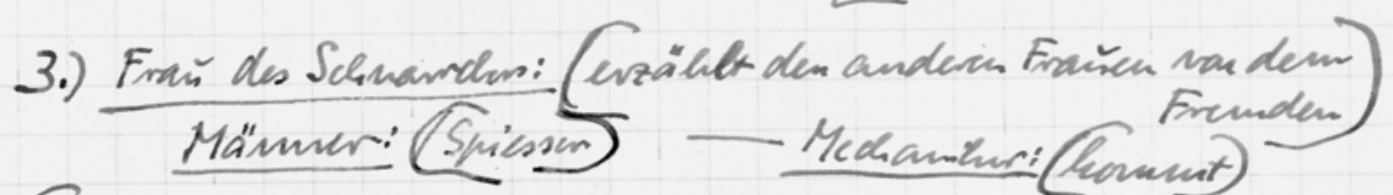

4.) Pendel. Yürigi scigt dem Ingenicion ch Pedal -

(4.)

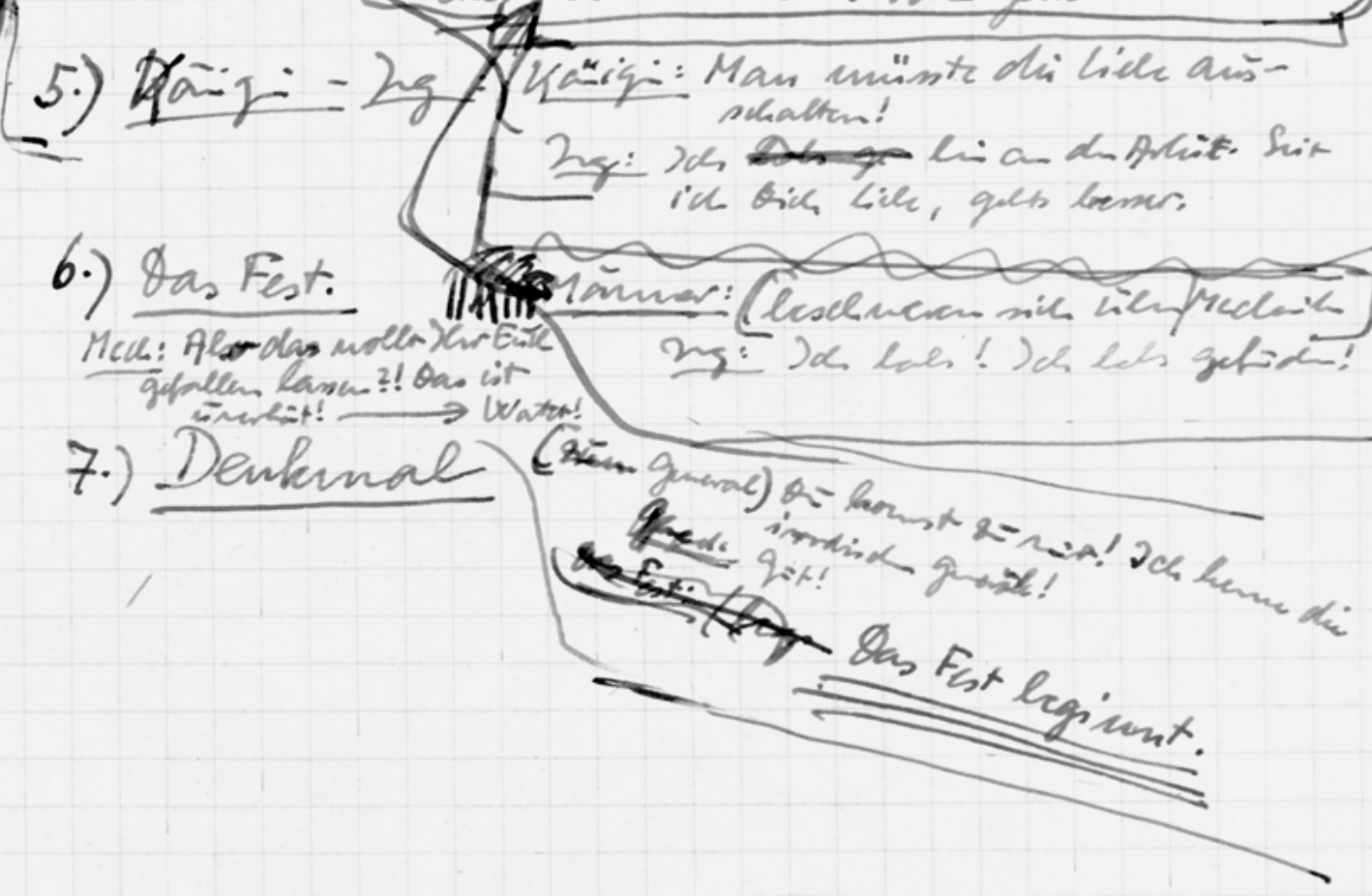

62 


\section{$\underline{\text { Himmelwärts }}$}

Zauberposse mit Gesang und Tanz.

1.) Denkmal

die Kënigi

2.) Wolke - (Palast) Pendel. - Palast

$(\mid[\mid$ Gericht $]$

3.) Frau des Schnarrchers: [erzählt den anderen Frauen von dem Männer: (Spiesser) - Mechaniker: (kommt) Fremden

4.) Pendel. Königin zeigt dem Ingenieur den Pendel

(4.) der bewacht wird vom General und Soldaten

5.) $\underline{\mathrm{D}} \mid \underline{\mathrm{K}} \underline{\underline{o ̈ n i g i n}}$ - Ing: Königin: Man müsste die Liebe ausschalten!

Ing: Ich bin an der Arbeit. Seit

Ich Dich liebe, gehts besser.

6.) Das Fest.

Männer. $([\mid[$ beschweren sich über|Mechaniker] Mech: Also das wollt Ihr Euch Ing: Ich habs! Ich habs gefunden! gefallen lassen?! Das ist unerhört! $\longrightarrow$ Wartet!

7.) Denkmal

(zum General) Du kommst zu mir! Ich kenne die irrdischen Generäle!

General: Gut!

Das Fest: (beg Das Fest beginnt. 
Strukturplan in sieben Bildern

ÖLA 3/W 50 - BS 41 a, Bl. 22

22

1.) Donlual.

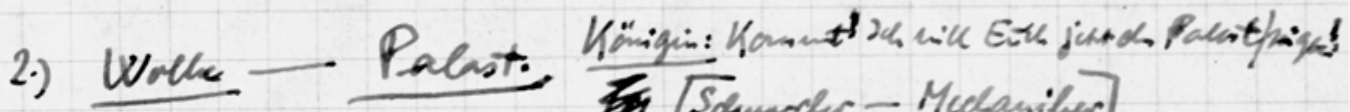

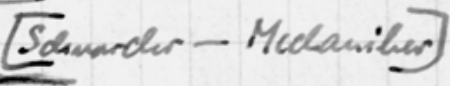

3.) Mecle., livligy der Erainens.

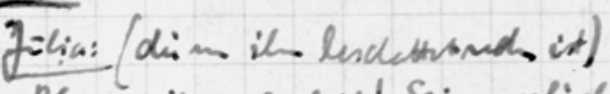

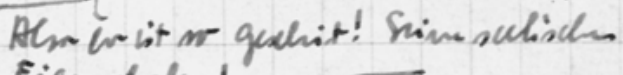
Eiguchattu!

4.) Das enige Pendel. (Mänuer lescewens sida)

5.) Med: (erfïhrt und der Enfichig) [Rurcte der firanien

6.) Das Fest.

7.) Dinuma.

64 


\section{1.) Denkmal.}

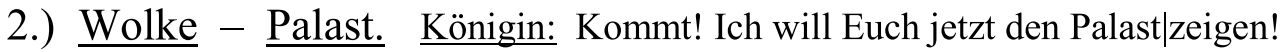
(M [Schnarcher - Mechaniker] 3.) $\underline{\underline{\text { Mech. }}}, \underline{\text { Liebling der Frauen. }} . \frac{\text { Julia: (die von ihm beschattet worden ist) }}{\text { Also er ist so gescheit! Seine seelischen }}$ Eigenschaften! - —

4.) Das ewige Pendel. (Männer beschweren sich)

5.) Mech. (erfährt von der Erfindung) [Revolte der Frauen]
6.) Das Fest .

7.) Denkmal. 
$\frac{\text { Himmel wästs }}{\text { Zariberposse mit Gesang ind Tanz in siclen Bildem. }}$

1.) Denkmalscenthiilling

2.) Wolke. Palast.

$\downarrow$

㔖

Ein Gevichtofale.

1. Frai" - T.Mamm

2. Fră" 2 .Mamu

(2)

Yönigin: (spindat Recht)

- 1

Köngin: (hamach shime Ingar. Ed Mech) Shat

Xur! Wir halon in der Vilminding der äumenn Nation alles enciar.! Wis halew Regu, Gailn, Th, Nacht - wri Cayge mir watlen, woom nir urlen! Wir halen nach

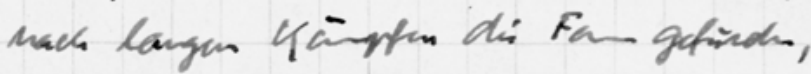

Wis halcu das

puepetrïume morlile

etfindur. line sosich Form - - aler, aler: dis inmen Nation? Was halen mir illumiñdar? Wis fremen lies cient sinsan, mir thalin lian mest - ind daum aree den Modvide, aluphe es aich sotche Sackes unchagit - Raifferria quing - aler soust - uni sind gearizor mit $X$ sad Xe sid mic dis thendet.' 


\section{Himmelwärts}

Zauberposse mit Gesang und Tanz in sieben Bildern.

\section{1.) Denkmalsenthüllung}

2.) Wolke .

$\underline{\text { Palast. }}$<smiles></smiles>

3.)

Ein Gerichtsfall.

1. Frau - 1. Mann

2. Frau - 2. Mann

$\circledast$

Königin: (spricht Recht)

$\circledast$ I

Königin: (hernach zum Ingen. und Mech) Seht

Ihr! Wir haben in der Überwindung der äusseren Natur alles erreicht! Wir haben

Regen, Gewitter, Tag, Nacht - wie lange wir wollen, wenn wir wollen! Wir haben nach nach langen Kämpfen die Form gefunden,

Wir haben das perpetuum mobile erfunden. eine soziale Form - - aber, aber:

die innere Natur? Was haben wir überwunden? Wir fressen uns nicht zusammen, wir lieben und treiben keinen Inzest - und dann auch den

Mordtrieb, obwohl es auch solche Sachen noch gibt - Raufereien genug -aber sonst - - wir sind genau so wie Ihr und Ihr seid wie die Urmenschen! 
Strukturplan in sieben Bildern (Fortsetzung)

ÖLA 3/W 50 - BS 41 a, Bl. 18

3.)

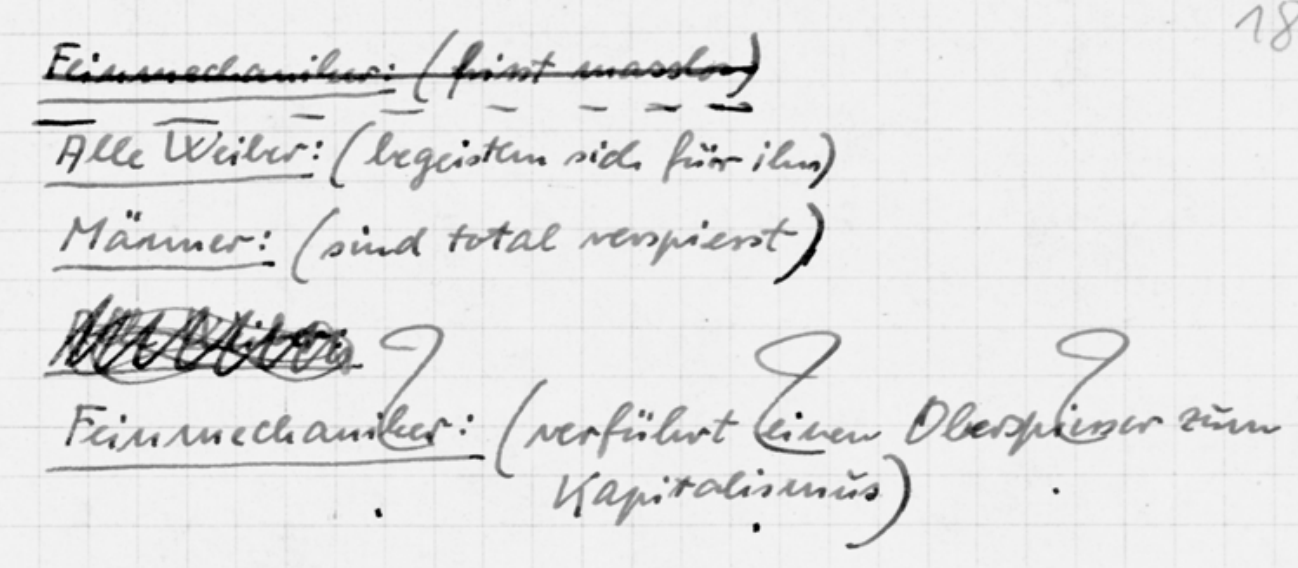

4.) Mämner hescheren sich hider Yörigin.

5.) Das Fest.

6.) Srivo des Einmechanilurs.

7.) Denimal.

68 


\section{3.) Feinmechaniker: (frisst masstes)}

Alle Weiber: (begeistern sich für ihn)

Männer: (sind total verspiesst)

Alle Weiber:

Feinmechaniker: (verführt einen Oberspiesser zum Kapitalismus)

4.) Männer beschweren sich bei der Königin.

(Erfindung des Ingenieurs.)

5.) Das Fest.

6.) Đas F $\underline{\text { Sturz des Feinmechanikers. }}$

7.) Denkmal. 


\section{Himunel wärts}

Zaüberposse mit Gesang ind Tanz in sichon Bilden.

1.) Denkmalsenthiilling

2.) Wolke.

- Palast.

$\downarrow$

㱛

Ein Gevichtofale.

1. Frain - T.Mamm

2. Frai" - 2. Mamm

2

Yönigin: (spindat Recht)

\section{- 1}

Köngin: (hamach thim Inoger. Ed Mech) Scht

Xhr! Whis halon in ders Vilminieding der ärmenn Natör alles enciar! Wist halew Regu, Gian, Th, Nadert - wai Cange min wallen, woume nir wrlen! Wir halen nach

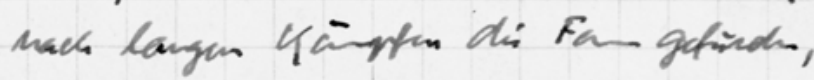

Wir halen das furpetionum morlile etfinden. line sosicle Form - - aler, aler:

dis inmen Nation? Was halen mis

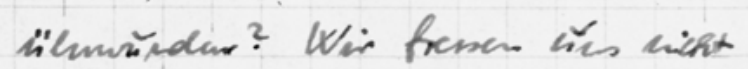

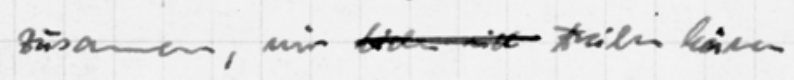
most - Ind dam ares den

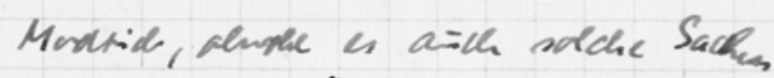
unchaint - Raiffercien quing - aler soust - uni sciedgeari or mie $x$ sid $X$ sid mic dis thende! 


\section{Himmelwärts}

Zauberposse mit Gesang und Tanz in sieben Bildern.

\section{1.) Denkmalsenthüllung}

2.) Wolke .

$\underline{\text { Palast. }}$<smiles></smiles>

3.)

Ein Gerichtsfall.

1. Frau - 1. Mann

2. Frau - 2. Mann

$\circledast$

Königin: (spricht Recht)

$\circledast 1$

Königin: (hernach zum Ingen. und Mech) Seht

Ihr! Wir haben in der Überwindung der äusseren Natur alles erreicht! Wir haben

Regen, Gewitter, Tag, Nacht - wie lange wir wollen, wenn wir wollen! Wir haben nach nach langen Kämpfen die Form gefunden,

Wir haben das perpetuum mobile erfunden. eine soziale Form - - aber, aber:

die innere Natur? Was haben wir überwunden? Wir fressen uns nicht zusammen, wir lieben und treiben keinen Inzest - und dann auch den

Mordtrieb, obwohl es auch solche Sachen noch gibt - Raufereien genug -aber sonst - - wir sind genau so wie Ihr und Ihr seid wie die Urmenschen! 
Strukturplan in sieben Bildern (Fortsetzung)

ÖLA 3/W 50 - BS 41 a, Bl. 19

3.) Der Finmuchamiker: (läng sich die maschinele 19 Alle Wailer: (sight war ilum begeistent) der Merre: (mit Hilte der Whiler, die wan ilhrew Männem vemacheänigt nerdew, emingte en ders Kapitalismins)

(b)

Merhes hapitalistisches Experiment.

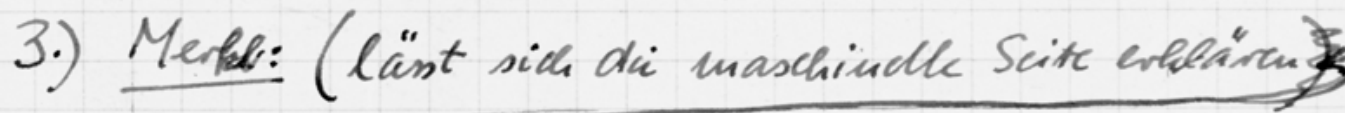
$\rightarrow$ äningent damm bapitalistiscle Wrimsche gegunieler cimem Brodigan;

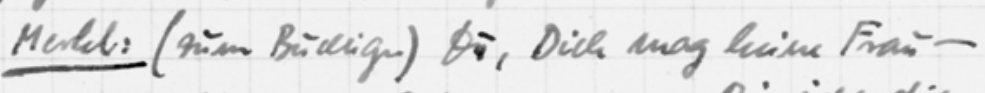

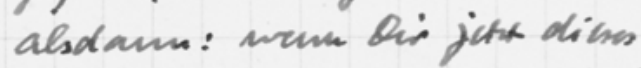

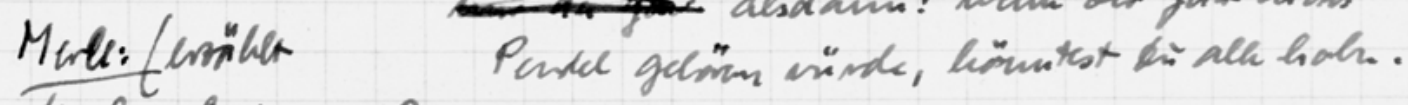

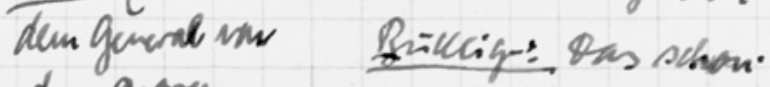

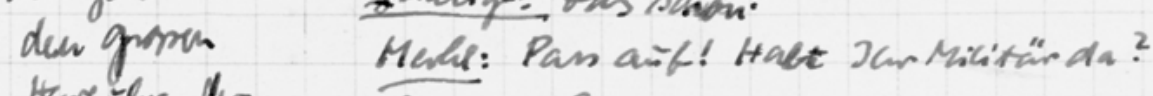

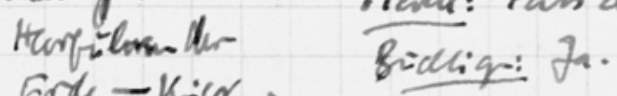

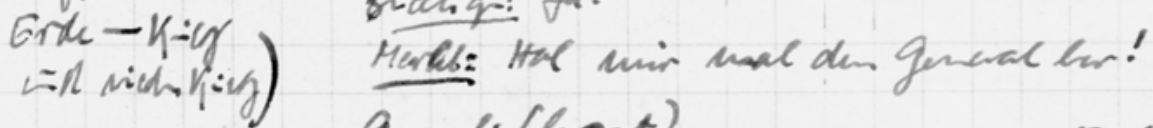
gead: (hont)

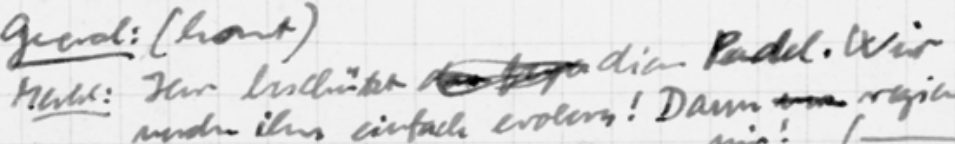

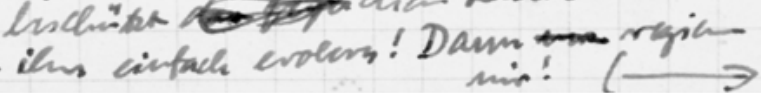

72 
3.) $\{\mathrm{M}\}$ Der Feinmechaniker : (lässt sich die maschinelle \{Sache\} Seite erklären)

Alle Weiber: (sind von ihm begeistert)

der Merkl: (mit Hilfe der Weiber, die von ihren Männern vernachlässigt werden, erringt er den Kapitalismus)

$\circledast$

\section{Merkls kapitalistisches Experiment. (Merkl wird eingesperrt)}

$\circledast$

3.) Merkl: (lässt sich die maschinelle Seite erklären) -

$\leftarrow$ äussert dann kapitalistische Wünsche gegenüber einem Buckligen;

Merkl: (zum Buckligen) Du, Dich mag keine Frau nur die \{ganz\} alsdann: wenn Dir jetzt dieses

Merkl: (erzählt Pendel gehören würde, könntest Du alle haben. dem General von den grossen Heerführern der Erde - Krieg Buckliger: Das schon.

Merkl: Pass auf! Habt Ihr Militär da?

Buckliger: Ja. und wieder Krieg)

Merkl: Hol mir mal den General her!

General: (kommt)

Merkl: Ihr beschützt das perpe dieses Pendel. Wir werden ihres einfach erobern! Dann regieren wir! ( 
Strukturplan in sieben Bildern (Fortsetzung)

ÖLA 3/W 50 - BS 41 a, Bl. 20

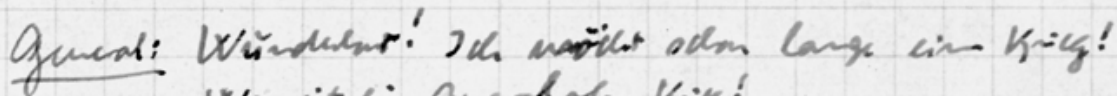
Was ist lia Guandohn Kiss!

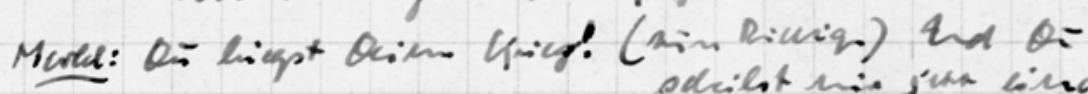

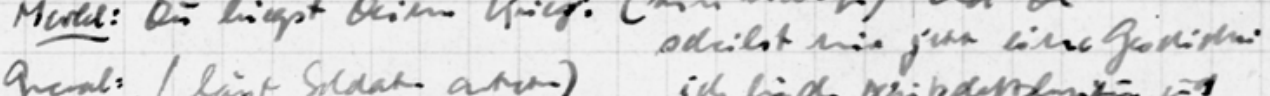

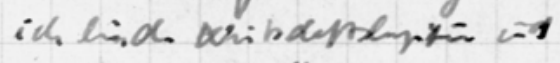
(Parare) min senorad!

wivide to bagnition a

Vrek: (strist sisand) oner siemand!

Mores: Eine vence Epache ist angelorods is Prhadien! Wis hah de Puder asd de Pedel Lot iñs! Wis maprech Eich Silge! Mdsacitur! Sellins wit dienen engrilig lde!

Vrels: Rrawr!

Eivs: Wino lrave? Was des da trier ist is Vulucha! Eis quaies Vuluchan!

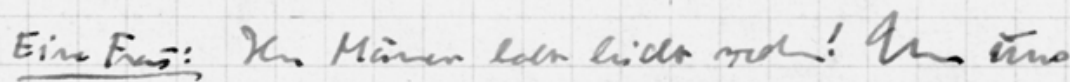
himut sich sinad! Esst nen co vider

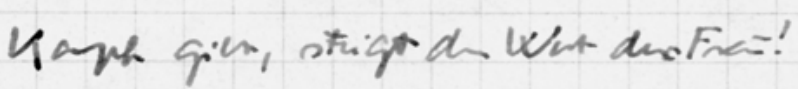

Stive: Oi Yäiq:!

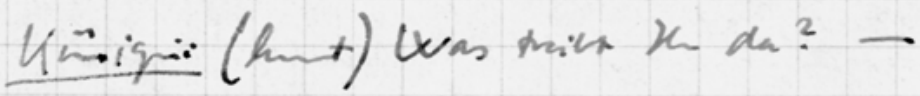
Ithst be es regene, General, dass wem

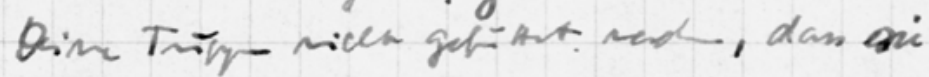

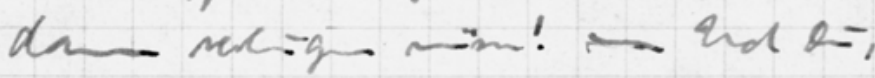
$B=u i g n$, mas trilut bich dan-?! Habt ux hisure Yoüh nogemen?!

Marde: (nid cingepeint) $>$

74 
General: Wunderbar! Ich möcht schon lange einen Krieg!

Was ist ein General ohne Krieg!

Merkl: Du kriegst Deinen Krieg! (zum \{Buckligen\}) Und Du

General: (lässt Soldaten antreten) schreibst mir jetzt eine Geschichte: ich bin der Wirtschaftskapitän und (Posaunen) sonst niemand!

Volk: (strömt zusammen) sonst niemand!

Merkl: Eine neue Epoche ist angebrochen in Arkadien!

Wir haben den Pendel und der Pendel hat uns!

Wir versprechen Euch Siege! Undsoweiter! Schluss mit diesem langweiligen Leben!

Volk: Bravo!

Einer: Wieso bravo? Was der da treibt ist ein

Verbrechen! Ein gemeines Verbrechen!

Eine Frau: Ihr Männer habt leicht reden! Um uns

kümmert sich niemand! Erst \&|wenn| es wieder

Kampf gibt, steigt der Wert der Frau!

Stimme: Die Königin!

Königin: (kommt) Was treibt Ihr da? -

Hast Du es vergessen, General, dass wenn

Deine Truppen nicht gefüttert werden, dass sie dann verhungern müssen! - Und Du, Buckliger, dass was treibt Dich dazu?! Habt Ihr unsere Kämpfe vergessen?!

Merkl: (wird eingesperrt) 
4.) Meral in Kälig.

(Oii Wiler hringen ilum Beimany - er

mind bequadigt iñd homment heañs - egile

sich Mins den Wiblen)

5.) Mänuer bescheveren sich hi den Käigion.

(Oi Enfinding)

6.) Das Fest.

7.) Denlemal. 
4.) Merkl im Käfig .

(Die Weiber bringen ihm Blumen) — er wird begnadigt und kommt heraus — ergibt sich nun den Weibern)

5.) Männer beschweren sich bei der Königin.

(Die Erfindung)

6.) Das Fest.

7.) Denkmal . 
Figurenliste

ÖLA 3/W 50 - BS 41 a, Bl. 24

Hirumelenints.

24

Pern:

Zigenew

Findoh

de Lisich Mäch

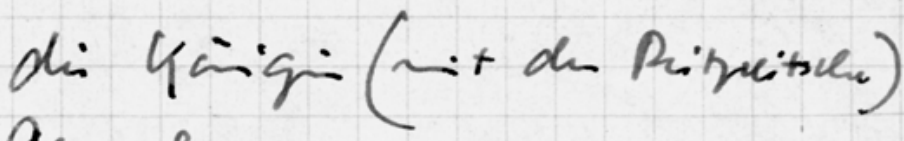

Gunal

den Schander

Jriat, new Fin =

der fiegrig

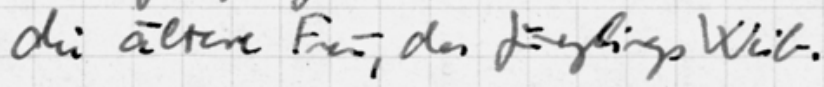

Mämuer

Whilur.

78 


\section{Himmelwärts.}

\section{Personen:}

Ingenieur

Feinmechaniker

das hässliche Mädchen

die Königin (mit der Reitpeitsche)

General

der Schnarrcher

Julia, eine Frau

der Jüngling

die ältere Frau, des Jünglings Weib.

Männer

Weiber. 


\section{郝}

Meold: (An̈m Schander) Jch Lale lin Gromes Mitgefinle unit Dori, wrilich aide schenandh!'

$\$$

Dilia: (2i Mule) Alur Dir hit gans-andens! Gars aders! Er schmamdes ja Her ĩd madr sust nices!!

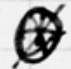




\section{$\circledast$}

Merkl: (zum Schnarrcher) Ich habe ein grosses

Mitgefühl mit Dir, weil ich auch schnarrch!

*

Julia: (zu Merkl) Aber Du bist ganz anders! Ganz anders! Er schnarrcht ja bloss und macht sonst nichts!!

$\circledast$ 
Notiz

ÖLA 3/W 50 - BS 41 a, Bl. 5

Mămuer:

diè sehr Dichew

2. Bild.

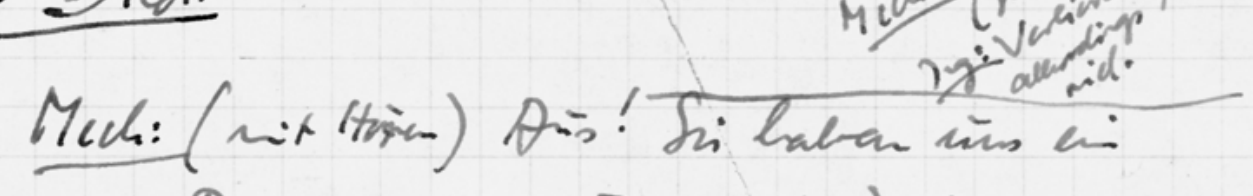
Dunhal goutht. Es bit kis falen her, das min algytlogan sind - mie

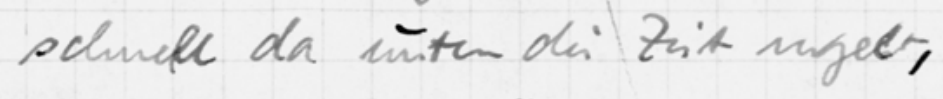
Muir sith, ab wais ant gesterm Gewerem.!

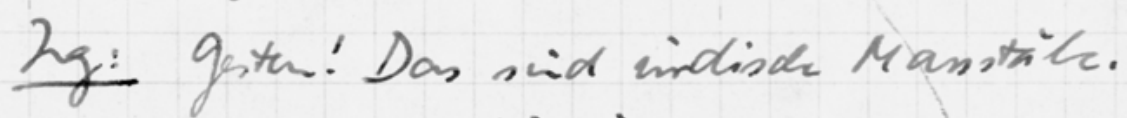
Whis sid hien ingedur, mas man $q^{\text {had }}$ indischen Mansoiten hicen

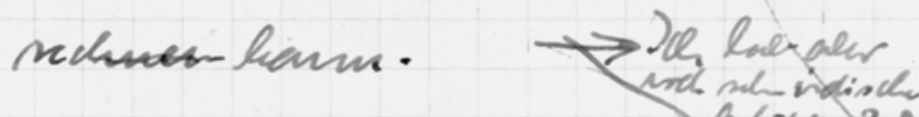
Men: Wor sidd mir lin cistared Mingl.

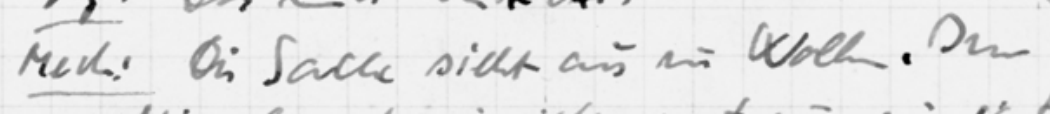

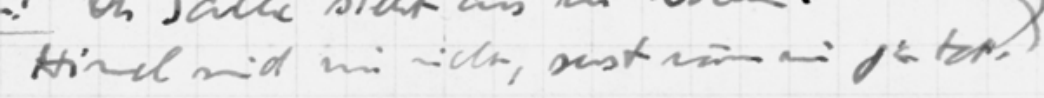

82 


\section{Männer:}

\section{der $\mid$ ie $\mid$ sehr Dicken}

$$
\left|E^{34}\right|
$$

2. Bild.

Ing: Auf die Erde Erde

können wir auf alle Fälle nichtmehr zurück.

Mech: Schad! Sehr schad!

(Stille)

Ing: Verlieren tun wir allerdings nicht

Mech: (mit Hörer) Aus! Sie haben uns ein viel.

Denkmal gesetzt. Es ist ein Jahr her, dass wir abgeflogen sind - wie schnell da unten die Zeit vergeht, mir ists, als wärs erst gestern gewesen!

Ing: Gestern! Das sind irrdische Massstäbe.

Wir sind hier irgendwo, wo man nach irrdischen Massstäben nicht rechnen kann.

Ich hab aber noch sehr irdische Gefühle. Z.B. Hunger.

Mech: Wo sind wir hier eigentlich.

Ing: Das weiss ich nicht.

Mech: Die Sache sieht aus wie Wolken. Im Himmel sind wir nicht, sonst wären wir ja tot. 
$\|$ 2. Bild.

Mech (mit Hörer) Aus! Sie haben uns ein Denkmal gesetzt. Es ist ein Jahr her, dass wir abgeflogen sind - wie schnell da unten die Zeit vergeht, mir ists, als wärs erst gestern gewesen!

5 ING Gestern! Das sind irrdische Massstäbe. Wir sind hier irgendwo, wo man nach irrdischen Massstäben nicht rechnen kann.

МесH Wo sind wir hier eigentlich.

ING Das weiss ich nicht.

MEch Die Sache sieht aus wie Wolken. Im Himmel sind wir nicht, sonst wären wir ja tot. Ich hab aber noch sehr irdische Gefühle. Z.B. Hunger.

ING Auf die ${ }^{\ulcorner}$Erde $^{\urcorner}$können wir auf alle Fälle nichtmehr zurück.

Mech Schad! Sehr schad!

(Stille)

ING Verlieren tun wir allerdings nicht viel.

\Abbruch der Bearbeitung\ 